absolutely tight at all pressures to which it is likely to be subjected.

Both spark wires were originally fused through the capillary stem, but from the oxides of nitrogen and carbon dioxide formed during the explosions an electrical connection was established sometimes between them and no spark was visible upon turning on the current. To obviate this, one fine spark wire, $w$, is fused through the capillary and the other, $v^{\prime}$, a platinum wire one and a half $\mathrm{mm}$. in diameter passes through a rubber fitting in the tube carrying the stop-cock up through the mercury to within two mm. of the other. A Mariotte bottle conveniently serves as a reservoir for the mercury and is connected with the pipette by quarter inch "pressure tubing."

It sometimes happens that no spark passes upon closing the circuit. This can be remedied by sucking water into the pipette and expelling it. Upon allowing it to stand all the moisture rises to the top and may be driven out.

The apparatus has been in use for four years, scores of explosions having been made with it. It may be obtained from the Ziegeler Electric Co., Franklin St., Boston.

\title{
ESTIMATION OF SULPHUR IN PYRITES.
}

\author{
BY G. Y.UNGE.
}

Received June 4,895 .

$\mathrm{N}$ this Journal Mr. Gladding replies to my remarks on his pre1 vious paper on the estimation of sulphur in pyrites. His comments would certainly not give to any reader, who had not my own paper before his eyes, a fair idea of its purport. He stales that I admit "that every modification proposed (by Gladding) is accurate in its nature, with one single exception." This conveniently passes over the fact that my "admission" had been saddled with the following addition, for which I had adduced ample experimental proof, "that not in a single case is Gladding's method more correct than mine, and that his modifications cannot be approved, as they greatly lengthen the time required for the analysis, without any corresponding advantage whatever." 
In one point, and that just relating to the principal novelty in Gladding's process, I declared him to be entirely wrong; viz., in his denial of the fact that it is very easy, by a special kind of washing, to avoid leaving any sulphur in the ferric hydroxide. This point is even more decidedly misrepresented by Gladding (of course, unintentionally) than the others. He says that I "admit that students in my own laboratory have sometimes failed to get out all the sulphur, through imperfect washing." I must really complain of this way of quoting my paper against myself, seeing that I immediately followed up the above by the express statement that such failures occurred in every case through having washed in the usual way, instead of the special way prescribed by me for that object; and that the same men have succeeded in every case after their attention had been drawn to this point. That beginners, like those I was speaking of, will make mistakes in most other analytical methods as well, until their attention is drawn to their having neglected some essential precautions, is too patent to be dwelled upon. Nor is it my business to find out why Mr. Gladding and his assistants have failed in a process which succeeds in the hands of all beginners in my laboratory, and which is practised by hundreds of chemists, none of whom have ever found any fault with it.

After such an involuntary "admission" of imperfect manipulation on Mr. Gladding's part, it is not possible to take the accuracy of his further results for granted which are contradictory to mine, concerning the differences between our two methods. I repeat that I have afforded in my paper, by a large number of comparative assays, complete proof that (except when the washing was intentionally interrupted before completion) absolutely the same results are obtained by both methods, mine, however, taking considerably less time than his. It seems to me perfectly unnecessary to repeat such proofs, since nothing new can possibly be brought out by it, and I must altogether decline to carry on further discussion with an opponent who treats my paper and work in the above-mentioned style, rewarding my studied fairness by construing it into all sorts of distorted "admissions," and not appreciating the perhaps exaggerated courtesy with which I had explained a decided error in his description of the 
decomposing-mixture as being perhaps due to a clerical mistake.

Mr. Gladding seems to have felt that it was necessary to bring heavier metal against me than his own assertions, and he therefore quotes a private communication from Prof. Richards, of Harvard College. He does not mention anything of Prof. Richards having sanctioned the rerbatim publication of the "private" communication. For my part, until the contrary is proved, I beg leave to doubt that Prof. Richards would publicly have used expressions accusing me of ignorance on a point "which has been known for a number of decades;". viz., the occlusion of barium chloride on the sulphate. Before doing so, he would have looked not merely at my last paper, but also on those I had previously written on the estimation of sulphur in pyrites. He would then have found that I have made researches on this subject for the last fifteen years, and that in my communications the previous work of Fresenius, concerning the difficulty of removing the barium chloride from the sulphate, is referred to as common knowledge. He would then not have misunderstood my last paper as denying that patent fact, which he has so thoroughly investigated in a paper published subsequently to mine. He would have acknowledged that I could not have meant anything else than claiming for my special process the greatest possible approach to truth, which would not be attained by preventing all and every occlusion of barium chloride, seeing that the solubility of the barium sulphate in the acid liquid must cause an error in the opposite direction. I am quite sure that Prof. Richards, like every other chemist, is aware of the fact that not one of our analytical separations is mathematically perfect, and that the best analytical methods are those in which unavoidable, but opposite errors are as nearly as possible balanced, and which thus gives a closer approximation to the truth than methods in which one of these errors is eliminated, but the compensating error in the opposite direction is allowed to remain. I have never claimed that the precipitate of barium sulphate obtained according to my method was absolutely devoid of barium chloride, no more than, in the opposite direction, that no barium sulphate whatever remained dissolved in the acid liquor; my claim has been that my method, by compensation of unavoidable errors, gives correct 
results. I need not, however, comment any further upon Prof. Richard's private letter to Mr. Gladding, since everything of importance in this controversy is settled by an authority which Prof. Richards will certainly approve of; viz., a passage in his own paper in the Zeitschrift fiur anorganische Chemie, $1895,8,4 \mathrm{I} 8$, which I translate verbatim:

"Moreover the observation is of interest, that the error caused by occlusion ordinarily just about compensates that caused by the solubility of the sulphate, so that the final corrected result is almost the same as the real weight. The average of the uncorrected results is 0.3215 in lieu of 0.3214 gram."

This, I think, disposes of the aid which Mr. Gladding has solicited from Prof. Richards in his attack upon me.

ZURICH, MAY 23, IS95.

\section{THE PERIODIDES. ${ }^{1}$}

\section{Bi Algert B. PREscott.}

Received A IIgust 12, 1895.

$T$ He HE periodides are remarkable as products of extreme additive combination, along with clearly cut crystalline form, distinct physical constants, and instances of rare optical power. They are easily reduced to normal iodides, containing for every atom of iodine firmly bound, one or more iodine atoms loosely bound; therefore they are often and not improperly termed superiodides. Professor Geuther used for them the term polyiodide, perhaps by reason of his view that all their iodine atoms are of equal valence in the molecule." Jörgenson designated them together as superiodides, though including within their structural type certain double polyiodides not understood to contain additive iodine. ${ }^{3}$ If these compounds contain, as their behavior has been interpreted to imply, for every atom of iodine that is linked to the base, a number of atoms of iodine linked only to iodine, they offer a striking example of the influence of a basal group upon iodine atoms to which it is not linked. The one iodine atom that is directly united to the nitrogen or other

1 Read at the meeting of the American Association for the Advancement of Science, Springfield, Aug. 30, I 895 .

2 A. Geuther, $1887: A n n$. Chem. (Liebig). $240,82$.

8 M. Jörgenson, 7869 : Ber. d. chem. Ges., 2, 465 . 